\title{
Unexpected disturbance of ultrastructure of porcine oocytes by very low concentrations of the solvent DMSO.
}

\author{
C. Campagna*, J.L. Bailey*, M.-A. Sirard* and P.Hyttel** \\ *Centre de Recherche en Biologie de la Reproduction, Department of Animal Sciences, Pavillon \\ Paul-Comptois, Université Laval, Québec, Canada, G1K 7P4. \\ **Department of Anatomy and Physiology, Royal Veterinary and Agricultural University, \\ Frederiksberg C, Denmark.
}

Dimethylsulfoxide (DMSO) is largely used for its cryoprotecting and solubilizing properties. Our team regularly uses $0.1 \%$ DMSO as a vehicle for the chemicals when we test the effects of an organochlorine mixture on oocyte maturation, fertilization and development. The main goal of this study was to determine the effects of this organochlorine mixture on porcine oocyte ultrastructure after $18 \mathrm{~h}$ of exposure during in vitro maturation (IVM). Since previous results showed that this mixture negatively affects oocyte maturation, fertilization and developmental competence[1], our hypothesis was that it would cause changes in the ultrastructure of the oocytes, especially regarding the endoplasmic reticulum (ER) and the mitochondria.

Porcine oocytes were matured in vitro $18 \mathrm{~h}$ in the presence or absence of the organochlorine mixture and the vehicle control (0.1\% DMSO). Samples were then embedded in agar before being processed for TEM (transmission electronic microscopy). Observation of oocyte ultrastructure was conducted using a Phillips CM 1000 electronic microscope and photographs were taken.

To our surprise, the DMSO control showed unexpected changes in the ultrastructure compared to the IVM solvent-free control. These changes were also apparent on the semi-thin sections (FIG 1). TEM analysis confirmed that the organochlorine treated oocytes did not differ much from the DMSO control with respect to their ultrastructure, but both greatly differed from the IVM solventfree control (FIG 2). Mild to drastic changes could be observed on lipid droplet appearance, the shape and number of vesicles, the ER network and the distribution of these organelles within the oocyte. Intriguingly, never in our previous studies have we observed functional effects caused by this small concentration of DMSO (TABLE 1; [1]).

Since the effects of the vehicle for our organochlorine mixture on the oocyte ultrastructure, we could not answer our hypothesis. In fact, we could not distinguish clear effects of the organochlorines on the ultrastructure compared to the DMSO effects. Nonetheless, this study emphasizes the importance of incorporating vehicle controls in all studies and experiments, even though it appears that the vehicle is not causing any effect to the test system. Since DMSO is largely used in the cryoprotection process of oocyte freezing, our results raise concerns about further effect of this chemical on the development and health of babies derived from cryoprotected eggs, event though no effect on pre-implantation development and implantation are actually observed. [2]

[1] C. Campagna et al., Biol. Reprod. 65 (2001) 554.

[2] This research was supported by the International Academic Mobility program of the Human Resources Development of Canada and the Toxic Substances Research Initiative program of Health Canada. Jytte Nielsen is greatly thanked for her skilled technical assistance. 


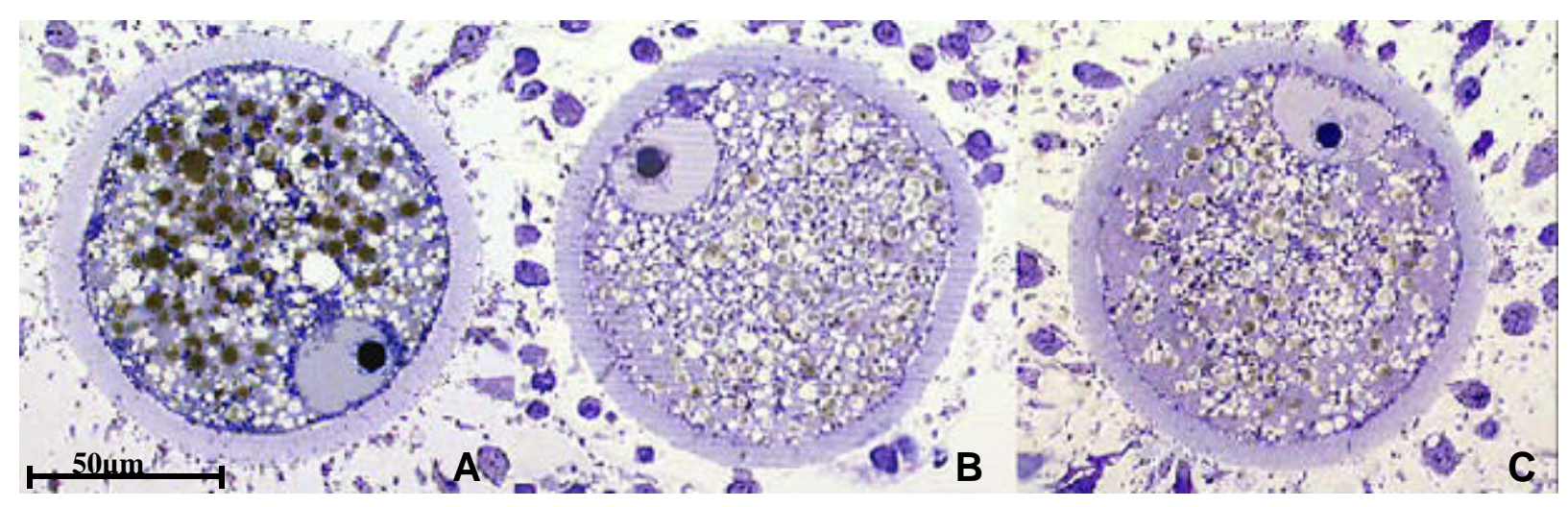

FIG 1. Light micrograph of porcine oocyte. A) IVM solvent-free control; B) 0.1\% DMSO control; C) organochlorine treated (0.1\% DMSO).
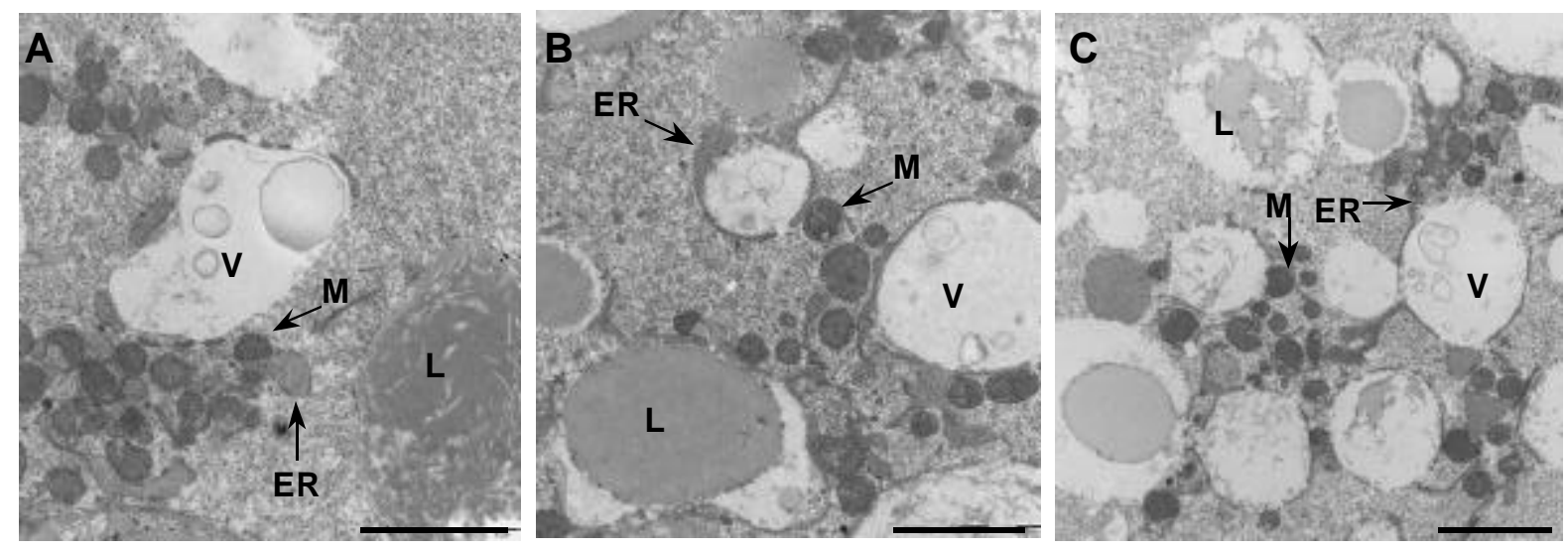

FIG 2. Electron micrograph from porcine oocyte. A) IVM solvent-free control; B) $0.1 \%$ DMSO control; C) organochlorine treated (0.1\% DMSO). L: lipid droplet; V: vesicle, M: mitochondria, ER: endoplasmic reticulum. Lines are $2 \mu \mathrm{m}$.

TABLE 1. Effect of $0.1 \%$ DMSO on porcine oocyte competence to maturation, fertilization and development. No significant differences were observed due to the presence of DMSO ( $p>0.05$ for all parameters).

\section{Parameters}

IVM solvent-free $\quad 0.1 \%$ DMSO control control

\begin{tabular}{lcc}
\hline \hline \% Perturbed maturation & $2.60 \pm 1.49$ & $4.18 \pm 1.49$ \\
\hline$\%$ Degenerated oocytes & $5.58 \pm 1.74$ & $3.14 \pm 1.74$ \\
\hline$\%$ Penetrated oocytes & $47.90 \pm 4.35$ & $32.90 \pm 4.35$ \\
\hline$\%$ Polyspermy & $30.66 \pm 10.22$ & $17.38 \pm 10.22$ \\
\hline$\%$ Developed to morula & $13.50 \pm 5.41$ & $18.80 \pm 4.42$ \\
\hline$\%$ Developed to blastocyst & $21.32 \pm 6.20$ & $16.32 \pm 5.06$ \\
\hline Number of cells per blastocyst & $43.50 \pm 4.93$ & $47.75 \pm 4.93$ \\
\hline
\end{tabular}

